\title{
Surgery for Hilar cholangiocarcinoma: the Newcastle upon Tyne Liver Unit experience
}

\author{
Abdullah Khalid Malik · Stuart Michael Robinson - Jeremy Jules French · Gourab Sen · Colin Hugh Wilson · \\ John Stotesbury Hammond - Steven Alan White - Derek Michael Manas
}

Received: 3 March 2019 / Accepted: 22 April 2019 / Published online: 17 May 2019

(C) The Author(s) 2019

\begin{abstract}
Summary
Background Hilar cholangiocarcinoma (HCCA) arises from the confluence of the common hepatic duct and has a poor prognosis. If resectable, an extended left (eLH) or right hemihepatectomy $(\mathrm{eRH})$ is usually required to provide oncological clearance. We reviewed
\end{abstract}

Dr. A. K. Malik, MB ChB, MRes $(\varangle)$ · S. M. Robinson, MB $\mathrm{ChB}, \mathrm{PhD} \cdot$ J. J. French, MB BS, MD · G. Sen, MB BS, MD . C. H. Wilson, MB BS, PhD · J. S. Hammond, BM BS, PhD . Prof. S. A. White, MB ChB, MD • Prof. D. M. Manas, MB BCh, MMed

Department of HPB and Transplant Surgery, Freeman Hospital, FreemanRoad, Newcastle upon Tyne, NE7 7DN, UK

abdullah.malik@nhs.net

S. M. Robinson, MB ChB, PhD

stuart.robinson@nuth.nhs.uk

J. J. French, MB BS, MD

jeremy.french@nuth.nhs.uk

G. Sen, MB BS, MD

gourab.sen@nuth.nhs.uk

C. H. Wilson, MB BS, PhD

colin.wilson@nuth.nhs.uk

J. S. Hammond, BM BS, PhD

john.hammond@nuth.nhs.uk

Prof. S. A. White, MB ChB, MD

steven.white@nuth.nhs.uk

Prof. D. M. Manas, MB BCh, MMed

derek.manas@nuth.nhs.uk

S. M. Robinson, MB ChB, PhD · C. H. Wilson, MB BS, PhD . Prof. S. A. White, MB ChB, MD - Prof. D. M. Manas, MB BCh, MMed

Institute of Cellular Medicine, Newcastle University,

4th Floor, William Leech Building, Medical School,

Framlington Place, Newcastle upon Tyne, NE2 4HH, UK outcomes for patients with HCCA managed at our centre.

Methods Electronic records of patients referred to our centre for HCCA were retrospectively reviewed. The Kaplan-Meier method was used to estimate overall survival (OS) with the log rank test used for significance $(p<0.05)$. A Cox regression was performed to ascertain factors that may influence survival.

Results 156 HCCA patients were identified (44 resected versus 112 non-resected). Resected patients had longer OS compared to non-resected patients (50.3 versus 9.8 months, $p<0.001)$. Patients who underwent an eLH $(n=15)$ had significantly longer OS at 3 years compared to eRH patients (67.7 vs. $42.1 \%$, respectively; $p=0.007$ ). An eLH was an independent predictor of survival (HR $0.43, p=0.04$ ). Lymph node positivity ( $n=23$, hazard ratio $1.72, p=0.027$ ) and the presence of microvascular invasion $(n=28$, hazard ratio $1.78, p=0.047$ ) were independent predictors of mortality. The frequency of lymph node positivity and microvascular invasion did not differ between eLH and eRH patients ( $p>0.05)$.

Conclusion Patients undergoing an eLH for HCCA have significantly better long-term outcomes compared to those undergoing eRH, independent of other pathological variables. The functional liver remnant (FLR) is usually smaller following eRH, resulting in a higher risk of post-operative liver failure. Combining CT volumetry with PVE may result in better prediction and optimisation of the FLR in the context of eRH for HCCA.

Novel findings An extended left hemihepatectomy is an independent predictor of survival; investigation into the precise interaction between left- and rightsided resections and pre- and post-embolization liver volume is warranted. 
Keywords Bile duct · Liver resection · Survival Biliary surgery $\cdot$ Biliary cancer

\section{Introduction}

Hilar cholangiocarcinoma (HCCA) is a malignant tumour arising from the confluence of the common hepatic duct into left and right branches. Pathologically, HCCA is known to spread longitudinally along the bile ducts, with concomitant perineural, microlymphatic and microvascular invasion [1, 2]. In early HCCA, haematological spread is rare; however, portal and pancreatic lymph node involvement is commonly seen, with one retrospective study reporting lymph node metastasis in $40 \%$ of resected patients [3].

HCCA has a poor prognosis, often due to locally advanced disease precluding curative treatment; patients with unresectable disease are known to have shorter survival compared to patients undergoing resection [2, 4, 5]. HCCA is generally considered unresectable in the presence of bilateral intrahepatic spread, peritoneal disease, involvement of the main portal vein, bi-lobar vascular involvement, and/or disease invading the contralateral hepatic artery or portal vein [6].

Operative and non-operative management of HCCA in both the curative and palliative settings is associated with significant risks of morbidity and mortality $[1,2]$. Resectable HCCA often requires an extended hemihepatectomy with en bloc resection of the caudate lobe to provide oncologic clearance of the tumour, which is associated with a significant mortality risk secondary to post-operative liver failure (PLF). Ipsilateral portal vein embolisation (PVE) improves operative outcomes for extended resections by inducing hypertrophy in the contralateral remnant liver pre-operatively, thereby reducing the risk of PLF [7, 8]. Biliary drainage is often necessary (as the majority of HCCA patients become jaundiced) to palliate unresectable patients and to relieve biliary obstruction in patients with potentially resectable disease $[1,7$, 9]. An extended right hemihepatectomy [10] results in a smaller functional liver remnant (FLR) compared to an extended left hemihepatectomy $(\mathrm{eLH})$, resulting in a significantly greater risk of PLF.

The aim of our study was to analyse the outcomes of all patients managed at our centre with HCCA during a 10-year period. This study follows up from a previous retrospective review [2] of the management of HCCA patients at our centre over an 8-year period (1995-2003), which was among the first documenting experiences in the management of HCCA in the UK [11].

\section{Methods}

\section{Patients}

The Newcastle hepatobiliary unit is a tertiary referral unit covering the north of England, serving a population of approximately 3.5 million. Patients with cholangiocarcinoma were retrospectively identified through hospital coding data over a 10-year period (February 2007 to March 2017), and electronic records were reviewed to detect patients with HCCA. Malignancy was confirmed for resected patients through pathology reports of resected specimens. In nonresected patients HCCA was confirmed through radiological imaging and serum CA 19-9 levels when elevated ( $>1000$ IU was regarded as diagnostic). In some cases HCCA was confirmed by FNA or by a biopsy taken at staging laparoscopy.

\section{Investigations, staging and non-operative management}

All patients referred to our centre with a probable diagnosis of HCCA underwent a focused multiphase contrast-enhanced CT examination including CT angiography using a multidetector CT unit to fully stage the disease. If further information was required to confirm the diagnosis or patients were considered potentially resectable, a dedicated MRI examination that included dynamic contrast-enhanced magnetic resonance angiography and MRI cholangiography was also performed. In most cases this was sufficient for providing details on vascular involvement of hilar vessels and liver volume. Tumours were radiologically staged according to the American Joint Committee on Cancer (AJCC) classification [12].

All jaundiced patients had percutaneous transhepatic biliary drainage (PTBD). Efforts were made not to cross the tumour where possible. For those patients who had mildly deranged liver function tests with a bilirubin concentration less than $70 \mu \mathrm{mol} / 1$ and who were considered resectable, patients were admitted a few days before surgery for PTBD to allow biliary decompression of the remnant liver. In a minority of cases, referring hospitals undertook PTBD or endoscopic retrograde cholangiography (ERCP). Endoscopic drainage performed at referring centres was discouraged. In patients with unresectable HCCA undergoing biliary drainage, self-expanding metallic stents were placed.

In resectable patients deemed to have an inadequate FLR volume (generally FLR $<30 \%$, depending on co-morbidities), PVE was performed to induce hypertrophy of the remnant segments.

All patients referred to our unit for management were discussed at the regional multi-disciplinary team (MDT) consisting of hepatobiliary surgeons, gastroenterologists, oncologists, radiologists and clinical nurse 
specialists prior to commencing definitive curative or palliative treatment.

\section{Surgical procedures and complications}

All patients with disease amenable to resection underwent a staging laparoscopy to exclude occult peritoneal disease. The definitive procedure was either an extended right hemihepatectomy with caudate lobectomy (for Bismuth-Corlette type 1, 2 and 3a HCCA) or an extended left hemihepatectomy with caudate lobectomy (for Bismuth-Corlette type 3b HCCA). Patients with potentially resectable type 4 disease were considered on a case-by-case basis to determine what type of resection was to be performed. A Roux-en-Y hepaticojejunostomy was used for biliary reconstruction following resection. Portal vein resections were performed if the tumour was encasing or directly involving the portal vein. Vascular continuity was restored by either a primary anastomosis or graft reconstruction with bovine pericardial patches. Pancreaticoduodenectomy was performed concomitantly in cases where the tumour extended into the pancreatic parenchyma and retro-pancreatic lymph nodes, e.g. stations 13 and 17. All patients underwent lymphadenectomy at the time of resection, usually involving stations 8,9 and 12. Patients found to be unresectable at laparotomy underwent segment 3 biliary-enteric bypass if the biliary tract had already been disconnected.

Patients experiencing post-operative bile leak (defined as drain bilirubin 3 times higher than serum bilirubin) were initially managed conservatively; the presence of fever (or any symptoms or signs of infection) resulted in the initiation of antibiotic therapy, tailored to bile cultures taken peri-operatively. PTBD was performed for bile leaks persisting after post-operative day 10 where obstruction was demonstrated on magnetic resonance cholangiography. Symptomatic bilomas were drained percutaneously under ultrasound guidance.

Complications were graded according to the Clavien-Dindo classification [13].

\section{Chemotherapy}

In line with existing evidence, we did not routinely offer adjuvant chemotherapy for patients who had undergone resection with curative intent. All patients with unresectable disease were offered palliative chemotherapy (typically gemcitabine/cisplatin) provided adequate biliary drainage (defined as serum bilirubin $\leq 27 \mu \mathrm{mol} / \mathrm{L}[\leq 1.58 \mathrm{mg} / \mathrm{dL}]$ ) had been achieved and patient performance status allowed. All patients developing disease recurrence following surgery are considered for palliative chemotherapy.

\section{Statistical analysis}

Statistical analysis was performed using SPSS version 22 (IBM Corp, Armonk, NY, USA). Overall survival (OS) and disease-free survival (DFS) were estimated using the Kaplan-Meier method, with comparisons made using the log-rank test. Continuous variables were compared using the t-test or Mann-Whitney U test. CA 19-9 elevated above the median value for the population was defined as high. Variables found to be statistically significant were included in a multivariate analysis using the Cox proportional hazards model. All statistical tests were twotailed, and a $p$-value $<0.05$ was considered significant. For comparison of survival between eLH and eRH, analysis was based on the resection intended based on pre-operative imaging and multi-disciplinary team discussion.

\section{Results}

A total of 156 patients with confirmed HCCA were managed at our centre. The male to female ratio was $1: 1$ and the median age at presentation for all HCCA patients was 68 years (42-91). For resected patients, the median age was 61 years $(42-71)$, compared with 72 years (42-91) for non-resected patients $(p<0.0001)$. There was no difference in median age when comparing patients who underwent eLH with patients who underwent eRH (60 vs. 61, $p>0.05$ ). A total of 115 patients (73.7\%) presented with jaundice, with the remainder presenting with right upper quadrant pain, weight loss, deranged liver function tests or incidentally on CT imaging. Following staging laparoscopy, 14 patients were excluded for resection due to peritoneal disease. A laparotomy for resection was performed in 44 patients (Table 1); 112 patients were deemed to have unresectable HCCA.

\section{Investigations}

All resected patients had a CT scan pre-operatively and $28(63.6 \%)$ patients underwent MR imaging as well; amongst the non-resected patients, 108 (96.4\%) underwent CT scanning and 37 (33.0\%) underwent MR imaging. The median CA 19-9 at the time of diagnosis in the resected group was $126 \mathrm{iU} / \mathrm{mL}$ compared to $647 \mathrm{iU} / \mathrm{mL}$ in the non-resected group $(p<0.0001)$. Median CA 19-9 at diagnosis did not differ when comparing patients undergoing eLH with patients undergoing eRH (103 vs. $147, p>0.05$ ).

\section{Table 1 Surgical procedures}

\begin{tabular}{|l|l|}
\hline Procedure & $N$ \\
\hline Extended right hemihepatectomy & 27 \\
\hline Extended left hemihepatectomy & 14 \\
\hline Extrahepatic bile duct resection & 1 \\
\hline Failed trial dissection (segment 3 biliary-enteric bypass) & 2 \\
\hline
\end{tabular}




\section{Pre-operative biliary drainage, post-operative biliary} drainage and PVE

In the resected group all 44 patients underwent preoperative biliary drainage, of whom 5 patients $(11.4 \%)$ were drained via ERCP and 39 patients (88.6\%) were drained via PTBD. Amongst non-resected patients a total of 167 PTBDs were performed; the median number of PTBDs per patient was $1(0-7)$, and 10 PTBDs failed $(6.0 \%)$. 22 non-resected patients (19.6\%) underwent ERCP of which 4 failed.

Post-resection, 11 patients (25\%) required drainage via PTBDs for post-operative bile leaks; a total of 22 PTBDs were performed, of which 3 failed.

A total of 11 patients (25\%) underwent PVE preoperatively to increase the FLR, of which all were of the right portal vein in patients undergoing an eRH.

\section{Surgical procedures and complications}

Amongst the 44 patients who underwent resection with curative intent (Table 1), 2 patients $(4.5 \%)$ were found to have unresectable HCCA at laparotomy, and surgical bypass was performed. A total of 15 patients underwent eLH and 29 patients underwent eRH. En bloc pancreatectomy was necessary in 7 patients (15.9\%), of which 5 were pylorus-preserving pancreaticoduodenectomies and 2 were total pancreatosplenectomies. Of the 7 patients requiring pancreatic resection, 1 patient was in the eLH group and 6 patients were in the eRH group. Portal vein resection was performed in 11 patients $(25.0 \%)$, with 1 patient requiring a graft reconstruction with an equine patch. Post-operative histopathology demonstrated vascular invasion in 4 patients $(36.3 \%)$ who underwent portal vein resection. Among the patients undergoing portal vein resection, 4 patients were in the eLH compared to 7 patients in the eRH group.

Post-operative complications are summarised in Table 2. Post-operatively, 35 patients (79.5\%) experienced complications, of whom 23 patients $(52.3 \%)$ demonstrated complications $\geq$ grade $3 \mathrm{a}$. The number of patients experiencing major post-operative complications ( $\geq$ grade $3 a$ ) was no different when comparing patients who underwent eLH with patients who underwent eRH (7 vs. 16, $p>0.05$ ).

\section{Resection, chemotherapy and survival}

In resected patients the 1-, 3- and 5-year OS was $92.0 \%, 56.0 \%$ and $38.0 \%$, respectively, with a median OS of 50.3 months $( \pm 32.2)$, with a 90-day all-cause mortality rate of $11.4 \%(n=5)$. In patients who underwent concomitant pancreatic resection $(n=7)$, the 90 -day mortality rate was $14.3 \%(n=1)$. This was significantly higher than median OS in non-resected patients (9.8 months \pm 3.8 ) with $1-, 3$ - and 5-year OS of $41.7 \%, 6.5 \%$ and $1.6 \%$, respectively ( $p<0.001$; Fig. 1$)$.
Table 2 Post-operative complications

\begin{tabular}{|l|l|}
\hline Complication & $n$ \\
\hline Grade I & 6 \\
\hline Wound infection & 2 \\
\hline Wound seroma & 1 \\
\hline Bile leak & 3 \\
\hline Grade II & 10 \\
\hline Pneumonia & 1 \\
\hline Bile leak & 3 \\
\hline Chyle leak & 1 \\
\hline Portal vein thrombosis & 1 \\
\hline Biliary sepsis & 3 \\
\hline Non-biliary sepsis & 1 \\
\hline Grade Illa & 9 \\
\hline Peri-hepatic collection & 4 \\
\hline Pleural effusion & 2 \\
\hline Pleuro-peritoneal fistula & 1 \\
\hline Empyema & 1 \\
\hline Bile leak & 1 \\
\hline Grade IIlb & 2 \\
\hline Bowel infarction & 1 \\
\hline Hepatic artery pseudoaneurysm & 1 \\
\hline Grade IVa & 8 \\
\hline Transient liver failure & 4 \\
\hline Acute kidney injury & 4 \\
\hline Grade IVb & 3 \\
\hline Sepsis & 2 \\
\hline Intra-abdominal haemorrhage & 1 \\
\hline Grade V/post-operative death & 5 \\
\hline Sepsis & 1 \\
\hline Intracranial haemorrhage & 1 \\
\hline Hepatic artery thrombosis & 1 \\
\hline Multi-organ dysfunction syndrome & 2 \\
\hline Total & \\
\hline
\end{tabular}

Patients undergoing eLH demonstrated significantly longer OS compared to patients undergoing eRH ( $p=0.007$; Fig. 2). The 1-, 3- and 5-year OS in patients undergoing eLH for HCCA was $100 \%, 67.7 \%$ and $58 \%$, respectively. In patients undergoing $\mathrm{eRH}$ for HCCA, the 1-, 3- and 5-year OS was $87.8 \%, 42.1 \%$ and $0 \%$, respectively. DFS was significantly longer in patients undergoing eLH $(p=0.006)$. The $1-, 3-$ and 5 -year DFS in the eLH group was $100 \%, 60.6 \%$ and $51.9 \%$, respectively. In the eRH group the $1-, 3-$ and 5 -year DFS was $66 \%, 21 \%$ and $0 \%$.

A total of 21 patients $(47.7 \%)$ demonstrated disease recurrence post-operatively. The patients with recurrence receiving chemotherapy demonstrated a longer median survival compared to patients with recurrence who were not offered chemotherapy (39.3 versus 26.7 months); however, this did not reach statistical significance $(p=0.813)$. Amongst non-resected patients, those who received palliative chemotherapy $(n=24)$ had a much longer median OS compared to 
Fig. 1 Overall survival (OS) in resected patients compared with non-resected patients. Median OS in resected patients was 50.3 months versus 9.8 months in non-resected patients $(p<0.0001)$

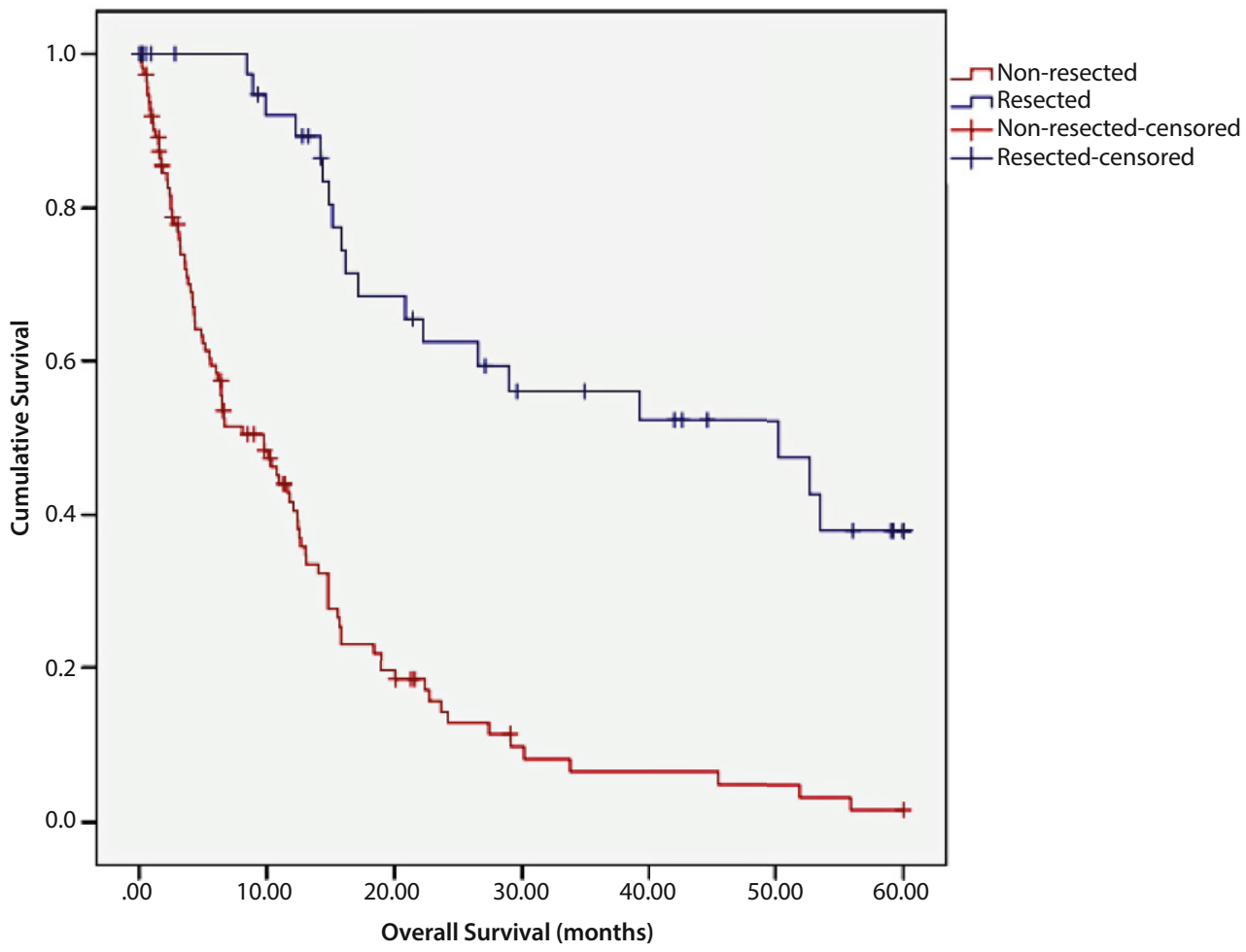

\begin{tabular}{|l|l|l|l|l|l|l|}
\hline No. at risk (months) & 0 & 10 & 20 & 30 & 40 & 50 \\
\hline Resected & 44 & 34 & 23 & 16 & 14 & 11 \\
\hline Non-resected & 112 & 45 & 17 & 6 & 4 & 3 \\
\hline
\end{tabular}

non-resected patients who did not receive chemotherapy $(15.5 \pm 4.3$ versus $6.4 \pm 1.6$ months, $p<0.0001)$.

\section{Prognostic factors for OS}

In univariate analysis (Table 3 ), significant predictors of shorter OS in the resected group were eRH ( $p=0.006)$, microvascular invasion $(p=0.025)$, lymph node positivity $(p=0.013)$. Resection margin (R0 $n=11)$, en bloc caudate resection $(n=36)$, en bloc pancreatectomy $(n=7)$, perineural invasion $(n=33)$, portal vein embolisation $(n=11)$ and portal vein resection $(n=11)$ did not impact on OS in univariate analysis. In multivariate analysis (Table 4), independent predictors of survival were eLH (HR $0.4195 \%$ CI $0.23-0.76 ; p=0.004)$, the absence of microvascular invasion on post-operative pathological examination (HR 0.56 95\% CI $0.31-0.99 ; p=0.047$ ) and negative lymph nodes (HR 0.58 95\% CI 0.36-0.94; $p=0.027$ ).

\section{Discussion}

Resection is the only treatment option offering longterm survival to patients with HCCA. In our series, an aggressive surgical approach conferred a significant survival benefit compared to those who were not resected. Among resectable patients, an extended left hemihepatectomy was an independent predictor of survival. This associated survival benefit is likely to be related to a larger FLR following eLH, resulting in a reduced risk of PLF compared to right-sided resections. Additionally, from an anatomical perspective, left-sided lesions may be more amenable to curative resection. The left hepatic duct is longer than the right, and divides into segmental branches later in its course compared to the right hepatic duct; consequently, left-sided lesions may be easier to access at resection. This is further supported by our observation that DFS is significantly longer in patients undergoing eLH.

Pre-operative PVE of the ipsilateral lobe and contralateral PTBD are strategies known to increase the FLR $[1,7,9,14]$. CT volumetry may help stratify patients into high- or low-risk categories for PLF, guiding pre-operative optimisation of patients with potentially resectable disease by identifying patients with potentially resectable disease who may benefit from PVE. Currently, no standardised pre-operative pathway exists for patients with potentially resectable HCCA; development and subsequent audit of a HCCA resection pathway may improve outcomes for such patients.

The need for portal vein resection and, when required, simultaneous en bloc pancreaticoduodenectomy did not significantly affect OS in our series. 
Fig. 2 Overall survival (OS) by type of resection. OS at 3 years in patients who underwent an extended left hemihepatectomy was $67.7 \%$ compared to $42.1 \%$ in patients who underwent an extended right hemihepatectomy $(p=0.007)$

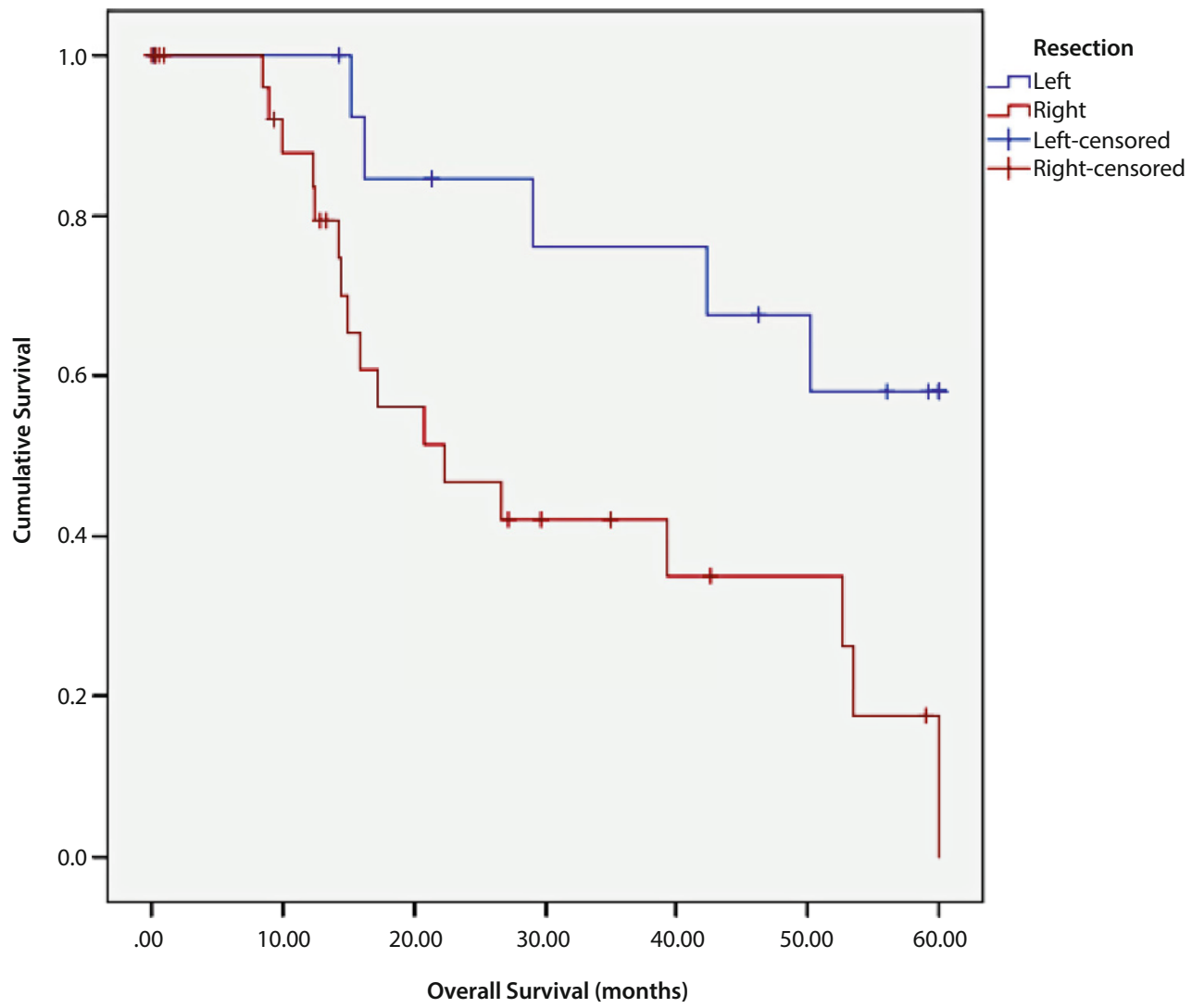

\begin{tabular}{|l|l|l|l|l|l|l|}
\hline No. at risk (months) & 0 & 10 & 20 & 30 & 40 & 50 \\
\hline Extended left & 15 & 14 & 11 & 9 & 9 & 7 \\
\hline Extended right & 29 & 21 & 12 & 7 & 5 & 4 \\
\hline
\end{tabular}

In a recent series that included a UK centre [15], combined pancreaticoduodenectomy (performed in 12 patients) was found to be a predictor of a higher rate of post-operative complications; however, no comment was made on its impact on OS. In our series, an R1 resection margin was not associated with shorter OS compared to an R0 margin, which has also been described by others in the UK [11]. Nevertheless this is not consistent across the board as microscopic tumour involvement at the resection margin can also be associated with worse rates of survival [5, 16-18]. As in other tumour types, this may be explained by discrepancies in histological reporting [19-21].

All patients in our series underwent portal lymphadenectomy, with clearance of the retro-pancreatic nodes if a pancreatectomy was also performed; lymph node involvement was associated with mortality in our series; therefore, en bloc resection may improve the efficacy of resection. The degree to which lymphadenectomy should be performed has not been standardised across centres. Extended lymphadenectomy is unlikely to confer any significant survival benefit [4]. A UK series of patients undergoing resection for HCCA [11] stated that all patients $(n=44)$ routinely underwent coeliac lymphadenectomy, of which 17 patients also underwent clearance of the para-aortic lymph nodes. The efficacy of performing such an extended lymphadenectomy is questionable, as suggested by others $[2-4,18,22]$. For example, patients having just a regional lymphadenectomy of the hepato-duodenal ligament have similar or superior 5-year survival rates compared to those having a more radical lymphanectomy, with the latter also having higher rates of morbidity. However, a study [23] investigating the efficacy of extended lymphadenectomy to the coeliac and para-aortic lymph nodes found that 5-year survival in patients with para-aortic lymph node metastasis was $15 \%$ and that extended lymphadenectomy did benefit some patients. However, another study [22] reported a 2-year survival rate of $0 \%$ in resected patients with lymph node metastasis beyond the hepato-duodenal ligament and it is therefore not surprising this study did not recommend extended lympadenectomy as routine.

Resection of an involved portal vein was carried out in 11 patients in our series. This has been shown to provide better oncological clearance [24], with 1- and 3 -year survival rates of $87 \%$ and $70 \%$, respectively [25]. 
Table 3 Univariate analysis of clinico-pathological variables that may influence OS in patients with HCCA undergoing resection

\begin{tabular}{|c|c|c|c|}
\hline Variable & $N$ & $\mathrm{HR}(95 \% \mathrm{Cl})$ & $p$-value \\
\hline \multicolumn{4}{|l|}{ Type of resection } \\
\hline eLH vs. eRH & 15 vs. 29 & $0.46(0.26-0.80)$ & 0.006 \\
\hline \multicolumn{4}{|c|}{ High CA 19-9 (>126iU/mL) } \\
\hline Low vs. high & 19 vs. 22 & $0.83(0.53-1.32)$ & 0.440 \\
\hline \multicolumn{4}{|l|}{$P V E$} \\
\hline No PVE vs. PVE & 33 vs. 11 & $0.87(0.52-1.46)$ & 0.600 \\
\hline \multicolumn{4}{|l|}{$T$-stage } \\
\hline T1/T2 vs. T3/T4 & 20 vs. 21 & $0.70(0.44-1.12)$ & 0.137 \\
\hline \multicolumn{4}{|l|}{ Microvascular invasion } \\
\hline Negative vs. positive & 16 vs. 28 & $0.53(0.31-0.92)$ & 0.025 \\
\hline \multicolumn{4}{|l|}{ Perineural invasion } \\
\hline Negative vs. positive & 11 vs. 33 & $0.64(0.33-1.26)$ & 0.200 \\
\hline \multicolumn{4}{|l|}{ Resection margin } \\
\hline R0 vs. R1 & 11 vs. 29 & $0.81(0.50-1.31)$ & 0.385 \\
\hline \multicolumn{4}{|c|}{ Lymph node involvement } \\
\hline No vs. yes & 21 vs. 23 & $0.55(0.35-0.88)$ & 0.013 \\
\hline \multicolumn{4}{|l|}{ Portal vein resection } \\
\hline No vs. yes & 33 vs. 11 & $0.90(0.54-1.53)$ & 0.703 \\
\hline \multicolumn{4}{|c|}{ En bloc pancreatic resection } \\
\hline No vs. yes & 37 vs. 7 & $0.75(0.44-1.27)$ & 0.282 \\
\hline \multicolumn{4}{|c|}{ Clavien-Dindo grade of complication $\geq$ grade $3 a$} \\
\hline Yes vs. no & 19 vs. 25 & - & 0.406 \\
\hline
\end{tabular}

Table 4 Multivariate analysis of clinico-pathological variables

\begin{tabular}{|c|c|c|c|}
\hline Variable & HR & $95 \% \mathrm{Cl}$ & $p$-value \\
\hline \multicolumn{4}{|l|}{ Resection } \\
\hline eLH vs. eRH & 0.41 & $0.23-0.76$ & 0.004 \\
\hline \multicolumn{4}{|c|}{ Microvascular invasion } \\
\hline No vs. yes & 0.56 & $0.31-0.99$ & 0.047 \\
\hline \multicolumn{4}{|c|}{ Lymph node involvement } \\
\hline No vs. yes & 0.58 & $0.36-0.94$ & 0.027 \\
\hline
\end{tabular}

Although portal vein resection has become somewhat routine in order to achieve oncological clearance, there is some evidence that portal vein resection may not provide any long-term benefit to patients with HCCA, due to increased morbidity $[18,26]$. Studies have reported similar survival rates for patients who underwent portal vein resection compared to patients in whom portal vein resection was not necessary [16, 18, 27]. A prospective study investigating the impact of portal vein resection on long-term outcome may be beneficial in establishing standard practice. In our series, no patients underwent arterial resection. There have been no major prospective studies investigating the efficacy of hepatic artery resection and reconstruction; however, anecdotal reports have suggested that arterial resection may be beneficial in selected patients [28-30] with arterial encasement/ involvement, although morbidity and mortality may be significantly higher. A Japanese study [31] evaluated outcomes of HCCA patients undergoing hepatic arterial resection and reported an operative mortality rate of $33 \%$ and a 3 -year survival rate of $0 \%$. This finding confirms that arterial resection is questionable. Arterial resection results in prolonged warm ischaemia of the liver parenchyma, which may contribute to the higher mortality observed in such patients. However, Nagino et al. reported a series of 50 patients with HCCA who underwent simultaneous portal vein and hepatic artery resection and reconstruction, with a 5-year survival rate of $30.6 \%$ [30]. Despite such a high 5-year survival rate, such patients experienced a lower survival compared to patients who did not require vascular resection. Therefore, arterial resection and reconstruction performed in high-volume centres may be more justifiable.

Pre-operative biliary drainage (PTBD) has become an essential part of the preparation of patients for resection. Although establishing adequate drainage can be associated with complications or mortality $[9,15]$, abandoning the practice would be unwise. Our practice is to ensure that the remaining lobe is well drained and the serum bilirubin is $<70 \mu \mathrm{mol} / \mathrm{L}(<4.09 \mathrm{mg} / \mathrm{dL})$. Standard practice in our centre for patients requiring pre-operative biliary drainage is to perform a PTBD rather than ERCP, as previous studies have demonstrated that ERCP is associated with a significantly greater risk of complications compared to PTBD [32], despite ERCP being more physiological.

Surgical resection of an obstructed liver is associated with poor outcomes due to coagulopathy, increased risk of cholangitis/biliary sepsis and increased risk of PLF secondary to liver atrophy [7]. Pre-operative biliary drainage of the FLR has been shown to improve outcome where the FLR is less than $30 \%$ [33]. A retrospective study [33] found that pre-operative PTBD resulting in an FLR $>30 \%$ was associated with fewer post-operative complications and better survival compared to patients with a FLR $<30 \%$ (which was found to be a strong predictor of morbidity and mortality). Patients with a FLR $<30 \%$ who underwent drainage experienced fewer complications and a higher survival rate.

However, a significant proportion demonstrated disease progression requiring a change in regimen. Studies investigating second-line chemotherapy regimens in advanced biliary tract malignancies are lacking but trials are currently ongoing (e.g. ABC06 trial investigating oxaliplatin and a modified de Gramont for patients previously treated with gemcitabine and cisplatin). Stereotactic body radiotherapy (SBRT) is another treatment modality that may offer longer survival to patients with unresectable HCCA. An American series retrospectively reviewed patients with cholangiocarcinoma who were treated with SBRT, demonstrating a median OS 17.0 months [34]. No randomised controlled trials investigating 
SBRT have been published; however, the ABC-07 trial is ongoing to investigate the feasibility of systemic chemotherapy (gemcitabine and cisplatin) plus SBRT.

The role for adjuvant and neoadjuvant chemotherapy in resectable HCCA is yet to be established, with clinical trials ongoing and yet to be reported. The BILCAP trial [35], investigating the efficacy of capecitabine for adjuvant chemotherapy in patients with cholangiocarcinoma, suggests that adjuvant chemotherapy extends median OS and should be routine practice. In our series, the longer OS for patients with recurrence who received chemotherapy compared to patients with recurrence who were not offered chemotherapy was not statistically significant; however, we continue to offer palliative chemotherapy to this group based on the evidence from the ABC-02 trial.

Our series is one of the few studies investigating the management of HCCA in a UK centre. We continue to advocate an aggressive surgical approach to treating patients with HCCA, as this offers the only hope of long-term survival. In our series, a left-sided resection was an independent predictor of survival. This is likely to be related to an increased risk of PLF (and further subsequent morbidity) following eRH due to a smaller FLR. Combining CT volumetry with PVE may result in better prediction and optimisation of the FLR in the context of eRH for HCCA, with a potential role for a standardised pre-operative HCCA resection pathway. Future prospective studies into the use of vascular resection and reconstruction, the timing of chemotherapy and the efficacy of SBRT would provide initial insight into establishing standard practices in the management of HCCA.

Author Contribution All authors contributed to the design and conception of the study, analysis and interpretation of data, and drafting and revising the final manuscript for submission. A.K. Malik, S.M. Robinson and D.M. Manas were responsible for acquisition of data.

\section{Compliance with ethical guidelines}

Conflict of interest A.K. Malik, S.M. Robinson, J.J. French, G. Sen, C.H. Wilson, J.S. Hammond, S.A. White and D.M. Manas declare that they have no competing interests.

Ethical standards The study was performed in accordance with the Declaration of Helsinki. It was non-interventional. Patient data were anonymized and retrospectively collected. Institutional board review was not required.

Open Access This article is distributed under the terms of the Creative Commons Attribution 4.0 International License (http://creativecommons.org/licenses/by/4.0/), which permits unrestricted use, distribution, and reproduction in any medium, provided you give appropriate credit to the original author(s) and the source, provide a link to the Creative Commons license, and indicate if changes were made.

\section{References}

1. Soares KC, KamelI, Cosgrove DP, HermanJM, PawlikTM.Hilar cholangiocarcinoma: diagnosis, treatment options, and management. HepatobiliarySurg Nutr. 2014;3(1):18-34.

2. Mansfield SD, Barakat O, Charnley RM, Jaques BC, O'Suilleabhain CB, Atherton PJ, et al. Management of hilar cholangiocarcinoma in the North of England: pathology, treatment, and outcome. World J Gastroenterol. 2005;11(48):7625-30.

3. Giuliante F, Ardito F, Guglielmi A, Aldrighetti L, Ferrero A, Calise F, et al. Association of lymph node status with survival in patients after liver resection for Hilar cholangiocarcinoma in an Italian multicenter analysis. JAMA Surg. 2016;151(10):916-22.

4. Rocha FG, Matsuo K, Blumgart LH, Jarnagin WR. Hilar cholangiocarcinoma: the Memorial Sloan-Kettering Cancer Center experience. J Hepatobiliary Pancreat Sci. 2010;17(4):490-6.

5. Jarnagin WR, Fong Y, DeMatteo RP, Gonen M, Burke EC, Bodniewicz BJ, et al. Staging, resectability, and outcome in 225 patients with hilar cholangiocarcinoma. Ann Surg. 2001;234(4):507-17. discussion 17-9.

6. Parikh AA, Abdalla EK, Vauthey JN. Operative considerations in resection of hilar cholangiocarcinoma. HPB (Oxford). 2005;7(4):254-8.

7. Belghiti J, Ogata S. Preoperative optimization of the liver for resection in patients with hilar cholangiocarcinoma. HPB (Oxford). 2005;7(4):252-3.

8. Ito F, Cho CS, Rikkers LF, Weber SM. Hilar cholangiocarcinoma: current management. Ann Surg. 2009;250(2):210-8.

9. Celotti A, Solaini L, Montori G, Coccolini F, Tognali D, Baiocchi G. Preoperative biliary drainage in hilar cholangiocarcinoma: Systematic review and meta-analysis. Eur J Surg Oncol. 2017;43(9):1628-35.

10. Bockhorn M, Uzunoglu FG, Adham M, Imrie C, Milicevic M, SandbergAA, etal. Borderline resectable pancreatic cancer: a consensus statement by the International Study Group of Pancreatic Surgery (ISGPS). Surgery. 2014;155(6):977-88.

11. Hidalgo E, Asthana S, Nishio H, Wyatt J, Toogood GJ, Prasad $\mathrm{KR}$, et al. Surgery for hilar cholangiocarcinoma: the Leeds experience. Eur JSurg Oncol. 2008;34(7):787-94.

12. Ganeshan D, Moron FE, Szklaruk J. Extrahepatic biliary cancer: new staging classification. World J Radiol. 2012;4(8):345-52.

13. Dindo D, Demartines N, Clavien PA. Classification of surgical complications: a new proposal with evaluation in a cohort of 6336 patients and results of a survey. Ann Surg. 2004;240(2):205-13.

14. Govil S, Bharatan A, Rammohan A, Kanagavelu R, Kaliamoorthy I, Reddy MS, et al. Liver resection for perihilar cholangiocarcinoma-why left is sometimes right. HPB (Oxford). 2016;18(7):575-9.

15. Kimura N, Young AL, Toyoki Y, WyattJI, Toogood GJ, Hidalgo $\mathrm{E}$, et al. Radical operation for hilar cholangiocarcinoma in comparable Eastern and Western centers: Outcome analysis and prognostic factors. Surgery. 2017;162(3):500-14.

16. Hemming AW, Mekeel K, Khanna A, Baquerizo A, Kim RD. Portal vein resection in management of hilar cholangiocarcinoma. J Am Coll Surg. 2011;212(4):604-13. discussion 13-6.

17. Cheng QB, Yi B, Wang JH, Jiang XQ, Luo XJ, Liu C, et al. Resection with total caudate lobectomy confers survival benefit in hilar cholangiocarcinoma of Bismuth type III and IV. Eur J Surg Oncol. 2012;38(12):1197-203.

18. Hoffmann K, Luible S, Goeppert B, Weiss KH, Hinz U, Buchler MW, et al. Impact of portal vein resection on oncologic 
long-term outcome in patients with hilar cholangiocarcinoma. Surgery. 2015;158(5):1252-60.

19. Menon KV, Gomez D, Smith AM, Anthoney A, Verbeke CS. Impact of margin status on survival following pancreatoduodenectomy for cancer: the Leeds Pathology Protocol (LEEPP). HPB (Oxford). 2009;11(1):18-24.

20. Jamieson NB, Foulis AK, Oien KA, Going JJ, Glen P, Dickson EJ, et al. Positive mobilization margins alone do not influence survival following pancreatico-duodenectomy for pancreatic ductal adenocarcinoma. Ann Surg. 2010;251(6):1003-10.

21. Ghaneh P, Kleeff J, Halloran CM, Raraty M, Jackson R, Melling J, et al. The impact of positive resection margins on survival and recurrence following resection and adjuvant chemotherapy for pancreatic ductal adenocarcinoma. Ann Surg. 2017;269(3):520-9

22. Kosuge T, Yamamoto J, Shimada K, Yamasaki S, Makuuchi M. Improved surgical results for hilar cholangiocarcinoma with procedures including major hepatic resection. Ann Surg. 1999;230(5):663-71.

23. Kitagawa Y, Nagino M, Kamiya J, Uesaka K, Sano T, Yamamoto $\mathrm{H}$, et al. Lymph node metastasis from hilar cholangiocarcinoma: audit of 110 patients who underwent regional and paraaortic node dissection. Ann Surg. 2001;233(3):385-92.

24. Neuhaus P, Jonas S, Bechstein WO, Lohmann R, Radke C, Kling N, et al. Extended resections for hilar cholangiocarcinoma. Ann Surg. 1999;230(6):808-18. discussion 19.

25. Neuhaus P, Thelen A, Jonas S, Puhl G, Denecke T, VeltzkeSchlieker W, et al. Oncological superiority of hilar en bloc resection for the treatment of hilar cholangiocarcinoma. Ann Surg Oncol. 2012;19(5):1602-8.

26. Chen W, Ke K, Chen YL. Combined portal vein resection in the treatment of hilar cholangiocarcinoma: a systematic review and meta-analysis. Eur J Surg Oncol. 2014;40(5):489-95.

27. Tamoto E, Hirano S, Tsuchikawa T, Tanaka E, Miyamoto $\mathrm{M}$, Matsumoto J, et al. Portal vein resection using the notouch technique with ahepatectomy for hilar cholangiocarcinoma. HPB (Oxford). 2014;16(1):56-61.
28. Matsuyama R, Mori R, Ota Y, Homma Y, Kumamoto T, Takeda K, et al. Significance of vascular resection and reconstruction in surgery for Hilar cholangiocarcinoma: with special reference to hepatic arterial resection and reconstruction. Ann Surg Oncol. 2016;23(Suppl4):475-84.

29. Wang ST, Shen SL, Peng BG, Hua YP, Chen B, Kuang M, et al. Combined vascular resection and analysis of prognostic factors for hilar cholangiocarcinoma. Hepatobiliary Pancreat Dis Int. 2015;14(6):626-32.

30. Nagino M, Nimura Y, Nishio H, Ebata T, Igami T, Matsushita $\mathrm{M}$, et al. Hepatectomy with simultaneous resection of the portal vein and hepatic artery for advanced perihilar cholangiocarcinoma: an audit of 50 consecutive cases. Ann Surg. 2010;252(1):115-23.

31. Miyazaki M, Kato A, Ito H, Kimura F, Shimizu H, Ohtsuka M, et al. Combined vascular resection in operative resection for hilar cholangiocarcinoma: does it work or not? Surgery. 2007;141(5):581-8.

32. Hameed A, Pang T, Chiou J, Pleass H, Lam V, Hollands M, et al. Percutaneous vs. endoscopic pre-operative biliary drainage in hilar cholangiocarcinoma-asystematic review and meta-analysis. HPB (Oxford). 2016;18(5):400-10.

33. Kennedy TJ, Yopp A, Qin Y, Zhao B, Guo P, Liu F, et al. Role of preoperative biliary drainage of liver remnant prior to extended liver resection for hilar cholangiocarcinoma. HPB (Oxford). 2009;11(5):445-51.

34. Mahadevan A, Dagoglu N, Mancias J, Raven K, Khwaja $\mathrm{K}$, Tseng JF, et al. Stereotactic body radiotherapy (SBRT) for Intrahepatic and Hilar cholangiocarcinoma. J Cancer. 2015;6(11):1099-104.

35. Primrose JN, Fox RP, Palmer DH, Malik HZ, Prasad R, Mirza D, et al. Capecitabine compared with observation in resected biliary tract cancer (BILCAP): a randomised, controlled, multicentre, phase 3 study. Lancet Oncol. 2019;20(5):663-73.

Publisher's Note Springer Nature remains neutral with regard to jurisdictional claims in published maps and institutional affiliations. 